\title{
Laparoscopic interventions in children on peritoneal dialysis
}

\author{
Maria Szczepańska ${ }^{1}$, Wojciech Korlacki², Krystyna Szprynger ${ }^{1}$, Andrzej Grabowski², Elżbieta Trembecka-Dubel ${ }^{1}$ \\ 1Department and Clinic of Paediatrics, Medical University of Silesia, Zabrze, Poland \\ 2Department and Clinic of Paediatric Surgery, Medical University of Silesia, Zabrze, Poland
}

Videosurgery and other miniinvasive techniques 2010; 5 (4): 152-157 DOI: 10.5114/wiitm.2010.18829

\begin{abstract}
Introduction: The benefits of peritoneal dialysis (PD) are multiple. However, it is connected with the risk of numerous complications, both infectious and non-infectious. Laparoscopic interventions in children on PD have seldom been presented.

Aim: To analyse laparoscopic interventions in 23 children (mean age $10.2 \pm 5.2$ years) treated with PD.

Methods: Performed procedures (30) included: diagnostic (explorative) laparoscopy, laparoscopic replacement of the catheter and laparoscopic recanalisation of occluded catheter.

Results: In 29 cases laparoscopy with reduced invasive procedures was completed. During surgery 5 catheters were removed. In 16 cases of catheter malfunction, omental wrap releasing, recanalization and/or reposition was done, including omentectomy in 9 cases. In 6 cases of acute abdomen symptoms, 3 adhesiolysis procedures in bowel obstruction, 2 appendectomies and in 1 case laparoscopic reduction of intussusception were done. There were no intraoperative complications. After surgery PD was continued in 19 children. Catheter survival rate was $261 \pm 297$ days. Laparoscopic surgery had no influence on peritoneal permeability or adequacy of PD treatment.

Conclusion: Laparoscopic technique is highly effective in children on PD especially related to catheter malfunction. It is an excellent option that enables restarting of PD shortly after the surgery.
\end{abstract}

Key words: peritoneal dialysis, children, laparoscopy

\section{Introduction}

Peritoneal dialysis is the method of choice in chronic dialysis treatment in children worldwide. The benefits of this method are multiple, including simplicity, low costs, better blood pressure control, fewer dietary restrictions, increased patients' independence, decreased transfusions, and no need for anticoagulation [1]. The disadvantages of PD are peritonitis, exit site infections, tunnel infections, hernias, dialysis fluid leakage, catheter mechanical obstruction with outflow complication and catheter dislodgement, and external cuff extrusion [2, 3]. Complications remain a significant cause of morbidity and periodic or permanent treatment failure despite the constant advances in catheter design and dialysis technique improvement. They also carry the risk for the patient to switch the method of treatment to haemodialysis.

Laparoscopy is currently used for catheter insertion and fixation in patients on peritoneal dialysis (PD) [4-7]. Good intraoperative visualization of the peritoneal cavity directly allows better evaluation and treatment of intra-abdominal pathology, sometimes early before the start of PD. Also cosmetic outcome plays an important role. For the better comfort of patients during laparoscopic surgery general anaesthesia is applied as insufflated carbon dioxide has an irritating influence on the peritoneal membrane. Laparoscopy is less invasive than laparotomy, and enables immediate postoperative PD continuation [8] 
and early enteral feeding application. Traditional conservative attempts to save the catheter with mechanical outflow obstruction are in most cases unsuccessful. The revolution in laparoscopic techniques has led to the development of various new methods to salvage the catheter [9-12].

Laparoscopic interventions in abdominal diseases or during complications in patients on PD, especially in children, have seldom been presented.

\section{Aim}

The aim of the current study was to analyse retrospectively our experience with laparoscopic interventions in children on PD.

\section{Material and methods}

Twenty-three children with renal failure on PD were treated by laparoscopic surgery from 1999 to 2008. They were operated on in the Clinic of Paediatric Surgery and remained in maintenance control at the Dialysis Division of the Clinic of Paediatrics in Zabrze, Medical University of Silesia in Katowice. Laparoscopic surgery is the method of choice in our centre in the management of children requiring surgical intervention. However, simple insertion and evacuation of a Tenckhoff catheter in the case of PD treatment termination is routinely performed by classical surgery. During the evaluation period only 1 child on PD with complications had open surgery due to the absence of a laparoscopic team. The study group included 15 girls and 8 boys, aged 3 months -18 years, mean $10.2 \pm 5.3$ years. The mean duration of dialysis to the point of surgical intervention was $489 \pm 711$ days ( 0 to 3220 days). The total number of patients on chronic PD treated in our centre was 93, on acute PD - 101 children.

The catheters were previously inserted by open surgery by the same team of surgeons. After catheter implantation children were treated in the dialysis unit and followed by a paediatric nephrologist. Automated peritoneal dialysis was applied with different treatment schedules, appropriate for particular patients' needs. In the evaluated group PD as the acute treatment was implemented in 3 patients (13\%), PD as the maintenance procedure in 20 patients (87\%).

The causes of renal failure in evaluated children included: chronic pyelonephritis -9 , chronic glomerulonephritis -5 , haemolytic-uraemic syndrome -4 , interstitial nephritis -2 , polycystic kidney disease -1 , familial nephronophtisis - 1 , amyloidosis -1 .
In 12 children straight Tenckhoff catheter, in 7 children self-positioning catheter, in 2 Swan-neck coil catheters were used. Two boys had a presternal catheter due to bilateral ureterocutaneostomy and Prune belly syndrome respectively. All catheters were double-cuff. The entry side was paramedian in 20 children, median in 3 children.

Thirty various laparoscopic procedures were performed in total. One boy was operated on 4 times, 2 boys and 2 girls twice each. The boy with the highest frequency of laparoscopic interventions had a presternal catheter which recurrently migrated.

Children were qualified for surgery after careful evaluation by a surgeon and paediatric nephrologist. In each case a surgeon experienced in laparoscopic technique performed the operation. The operation started after complete drainage of the peritoneal cavity. Pneumoperitoneum was installed by a Veress needle or via the dialysis catheter (if not occluded) with intraabdominal pressure of $8-12 \mathrm{mmHg}$ according to the patient's age. Videoscopic monitoring was performed via the $5 \mathrm{~mm}$ or $10 \mathrm{~mm}$ port inserted near the umbilicus. Subsequently the number and the area of placement of operative ports were stated, knowing the pathology and required surgery. Most often two operative $5 \mathrm{~mm}$ ports were applied. After the operation the peritoneal cavity was deflated and carbon dioxide removed, ports were evacuated and sutures created.

Performed procedures included: diagnostic (explorative) laparoscopy, laparoscopic replacement of the catheter and laparoscopic recanalisation of the occluded catheter (adhesiolysis, omentectomy, omental wrap releasing, omentopexy). The laparoscopic procedures lasted 50 min on average. Postoperative pain was controlled by intravenous analgesics.

Statistical analysis was performed using Statistica 8.0 PL (StatSoft, Inc 2008). Catheter survival was evaluated using Kaplan-Meier life table survival analysis. One-way ANOVA was applied to estimate the significance of differences between adequacy indices and permeability before and after surgery. The significance level was defined as $P<0.05$.

\section{Results}

The percentage of operations in relation to the duration of PD before surgery is shown in Figure 1. Fifty percent of interventions were early (less than 5 months) after initial catheter insertion. 


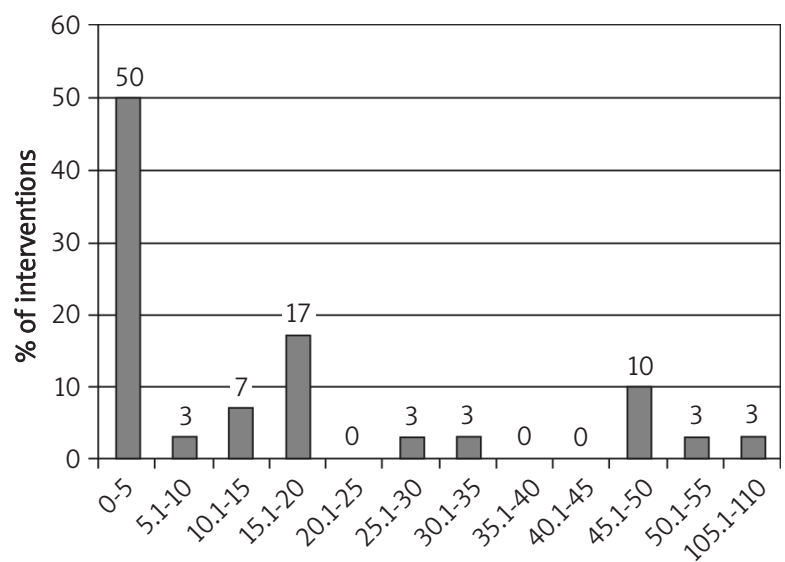

Figure 1. Duration of peritoneal dialysis treatment in study group before surgery

The causes of laparoscopy included: drainage problems in 16 cases (53.3\%), symptoms of ileus in $6(20 \%)$, peritonitis resistant to pharmacothe-
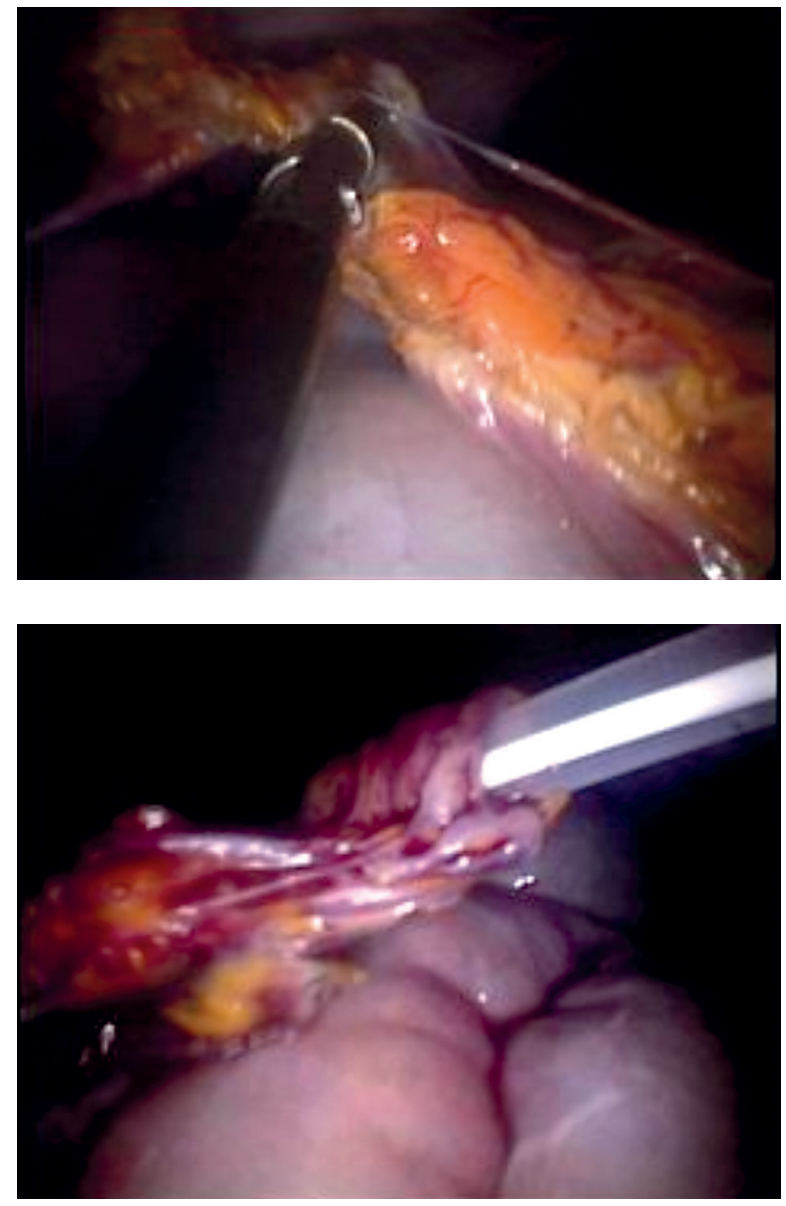

rapy in 6 (20\%), haemoperitoneum in 1 (3.3\%), and scheduled laparoscopic Toupet fundoplication in $1(3.3 \%)$.

In 29 cases laparoscopy with reduced invasive procedures was completed. In one girl the attempt of laparoscopy could not be continued due to massive intraperitoneal adhesions - the catheter was removed and haemodialysis applied. During surgery 5 catheters were removed. Four of them were replaced.

In 16 cases of catheter malfunction, omental wrap releasing, recanalization and/or reposition was done, including omentectomy in 9 cases (Figure 2). In 6 cases of acute abdomen symptoms, 3 adhesiolysis procedures in bowel obstruction, 2 appendectomies, and in 1 case laparoscopic reduction of intussusception were done. In 5 cases of prolonged peritonitis diagnostic laparoscopy with peritoneal biopsy was performed. There were no intraoperative complications.
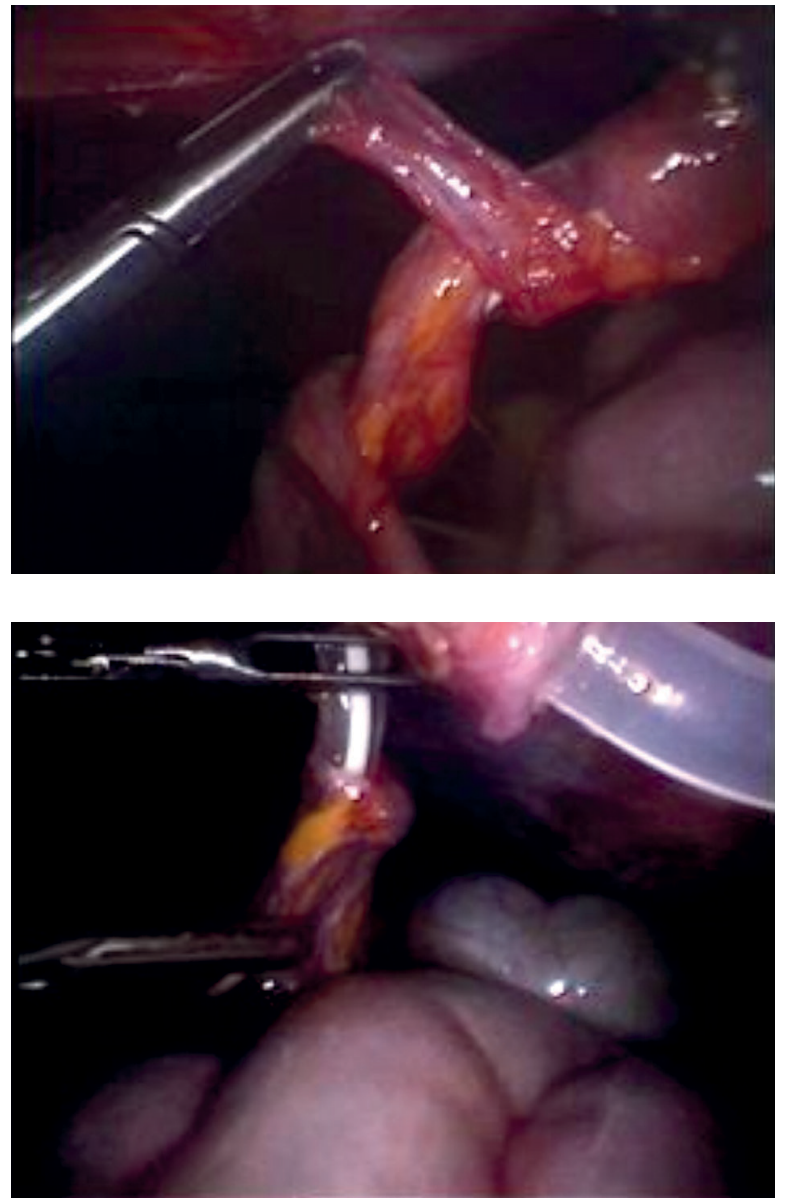

Figure 2. Partial omental resection using LigaSure device. Steps of catheter salvage - removing omentum wrapped around the catheter 
The most serious complications after laparoscopy were: respiratory failure (3 cases - 10\%), dialysate leakage (3 cases $-10 \%)$, haemoperitoneum ( 1 case $3.3 \%$ ), and serious metabolic disorders (2 cases $6.7 \%$ ). All above-mentioned complications were present in children with BMI lower than $21 \mathrm{~kg} / \mathrm{m}^{2}$. Other complications included fever, transient eosinophilic peritonitis and hypervolaemia. Figure 3 shows complications during laparoscopic surgery in relation to the previous dialysis duration.

After surgery PD was continued in 19 children. Fluid exchanges were started a few hours after surgery. In children on acute PD in 2 children renal function recovered and 1 child died.

Catheter survival rate was $261 \pm 297$ days. Six catheters were subsequently removed during transplantation in another centre, and 5 were still used at the end of study observation in November 2008. Three children died from causes not related to catheter malfunction. One child was transferred to haemodialysis due to ultrafiltration failure. Kaplan-Meier analysis results of catheter survival are shown in Figure 4.

In patients on maintenance PD analysis of membrane function and dialysis adequacy was done in comparison to peritoneal function before and after surgery.

Before surgery the mean value of KT/V was 2.58 \pm 0.57 and the mean value of creatinine clearance was $67.06 \pm 24.67 \mathrm{l} /$ week $/ 1.73 \mathrm{~m}^{2}$. After surgery the mean value of $\mathrm{KT} / \mathrm{V}$ was $2.65 \pm 1.02$ and the mean value of creatinine clearance was $65.51 \pm 32.34 \mathrm{l} /$ week $/ 1.73 \mathrm{~m}^{2}$.

There was no difference in KT/V and creatinine clearance between the values before and after surgery (KT/V $p=0.8070$, creatinine clearance $p=0.8722$ ).

Figure 5 shows the comparison of peritoneal permeability evaluated by PET test. No difference in peritoneal permeability before and after laparoscopic intervention was detected $(p=0.5201)$.

\section{Discussion}

The laparoscopic attempt of correction of malfunctioning PD catheters by laparoscopy was done for the first time by Wilson and Swartz [13]. The next step was the placement of Tenckhoff PD catheters in the 1980s [14]. Application of this technique in PD patients, especially children, requires a surgeon experienced in laparoscopy to minimize the complications during the operation and in the postoperative period $[12,15]$.

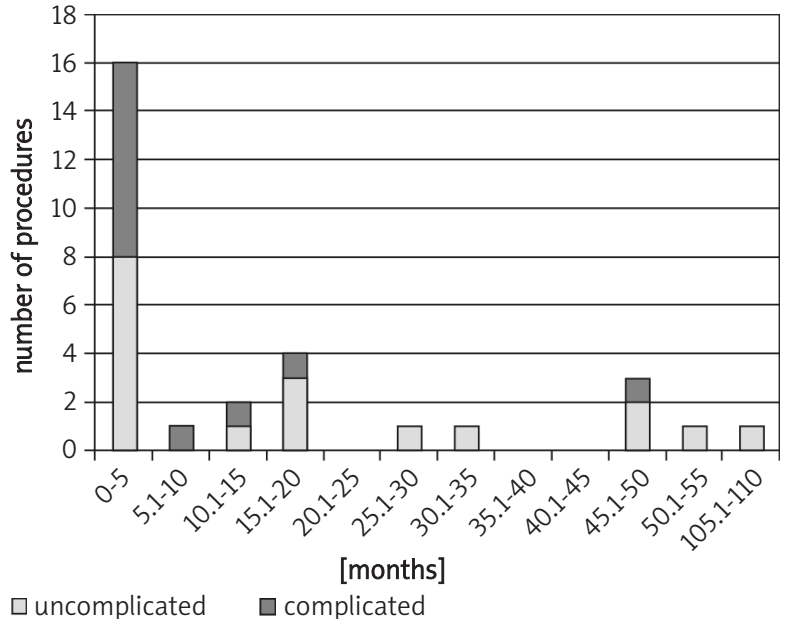

Figure 3. Complication during laparoscopic surgery in relation to dialysis duration

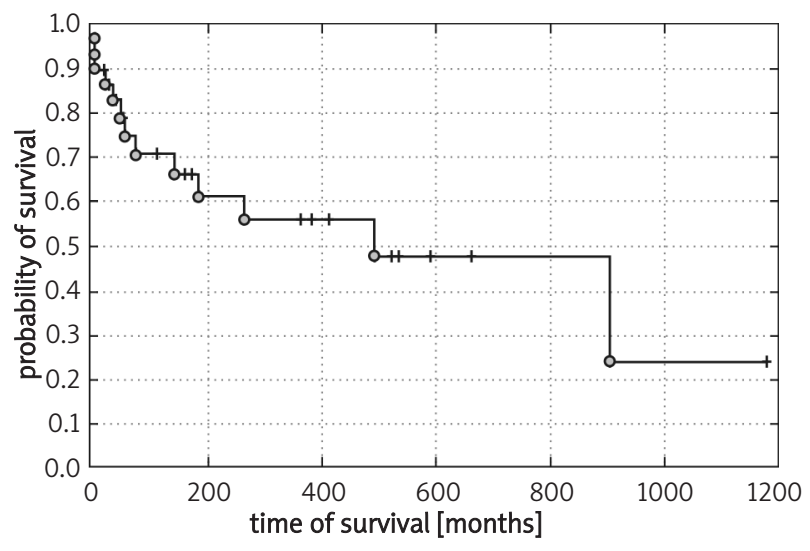

o failed catheters + functioning catheters

Figure 4. Catheter survival after laparoscopic surgery

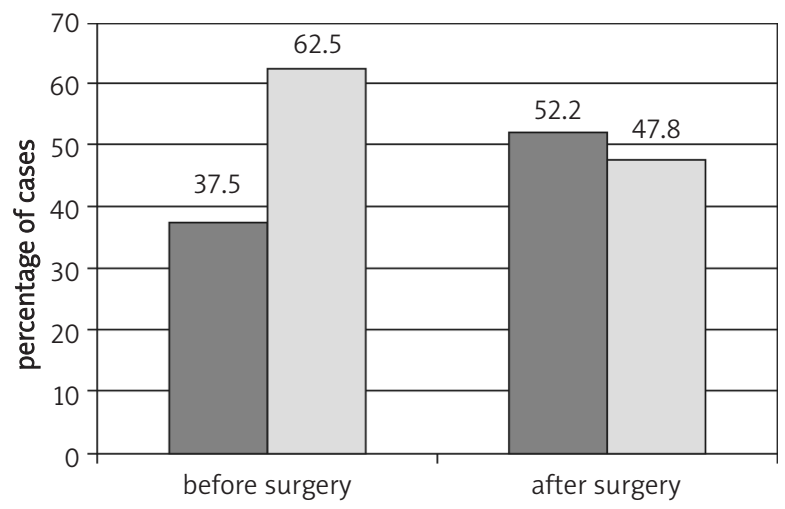

$\square \mathrm{L}+\mathrm{LA} \quad \square \mathrm{H}+\mathrm{HA}$

Figure 5. Peritoneal permeability evaluated by PET test 
Nowadays laparoscopy is moving toward microinvasiveness. Operations in children could be performed with less trauma and better end results even in more complicated procedures [16, 17]. Instruments have been made smaller and thinner, which creates the possibility of small incisions $[18,19]$. Minimally invasive laparoscopic technique carries lower risk of dialysate leakage, as well [11]. In our series this problem (leakage from the catheter site) was present in $10 \%$ of children, which is a favourable result.

Direct intraoperative view into the failed catheter allows for better recognition of the underlying disorder and for application of the proper manoeuvre to restore its function in mechanical outflow obstruction [20]. Laparoscopy can also be applied in PD children with poor clinical condition because of its safety properties. In our group $50 \%$ of interventions were in the first 5 months after the initial catheter implantation, 1 in a small newborn requiring intensive care support. Additionally, complications after laparoscopic surgery were present in all children dialysed for less than 10 days.

Laparoscopy is also useful in the case of presternal catheters, when reposition with a stiff wire without surgery is impossible. The described boy with such a catheter had 4 laparoscopic interventions because of permanent catheter migration. He was successfully transplanted at the end with this same catheter functioning for 2.25 years.

As the main cause of catheter malfunction is omental wrap, we try to perform partial omentectomy as a routine procedure at the time of catheter insertion in chronic PD patients. However, in 30\% of cases subsequent omental wrap releasing (Figure 2) and omentectomy was necessary.

We showed that catheter salvage by this method is of great benefit for children. One year after the surgery more than $55 \%$ of catheters were still functioning in our patients and the 2-year survival was around $48 \%$. Laparoscopy also preserved proper function of the peritoneum. In all children continuing PD adequacy measured by creatinine clearance and KT/V after the intervention was the same as before the laparoscopy. Similarly, peritoneal permeability evaluated by PET test showed no decline after the surgery.

In summary, laparoscopic technique is highly effective for management of various surgical problems in children on PD especially related to catheter malfunction. It is an excellent option for surgical interventions in the maintenance PD group, causing only minimal abdominal wall and peritoneum injury, which enables restarting of PD shortly after the surgery. Laparoscopic surgery has no influence on peritoneal permeability or adequacy of PD treatment.

\section{References}

1. Lessin MS, Luks FI, Brem AS, et al. Primary laparoscopic placement of peritoneal dialysis catheters in children and young adults. Surg Endosc 1999; 13: 1165-7.

2. Macchini F, Valade A, Ardissino G, et al. Chronic peritoneal dialysis in children: catheter related complications. A single centre experience. Pediatr Surg Int 2006; 22: 524-8.

3. Rees L. Long-term peritoneal dialysis in infants. Perit Dial Int 2007; 27: 180-4

4. Ashegh H, Rezaii J, Esfandiari K, et al. One-port laparoscopic technique for placement of Tenckhoff peritoneal dialysis catheters: report of seventy-nine procedures. Perit Dial Int 2008; 28: 622-5.

5. Daschner M, Gfrörer S, Zachariou Z, et al. Laparoscopic Tenckhoff catheter implantation in children. Perit Dial Int 2002; 22: 22-6.

6. Mattioli G, Castagnetti M, Verrina E, et al. Laparoscopic-assisted peritoneal dialysis catheter implantation in pediatric patients. Urology 2007; 69: 1185-9.

7. Stringel G, McBride W, Weiss R. Laparoscopic placement of peritoneal dialysis catheters in children. J Pediatr Surg 2008; 43: 857-60.

8. Varela JE, Elli EF, Vanuno D, et al. Mini-laparoscopic placement of a peritoneal dialysis catheter. Surg Endosc 2003; 17: 2025-7.

9. Brandt CP, Ricanati ES. Use of laparoscopy in the management of malfunctioning peritoneal dialysis catheters. Adv Perit Dial 1996; 12: 223-6.

10. Kim HJ, Lee TW, Ihm CG, et al. Use of fluoroscopy-guided wire manipulation and/or laparoscopic surgery in the repair of malfunctioning peritoneal dialysis catheters. Am J Nephrol 2002; 22: 532-8.

11. Numanoglu A, Rasche L, Roth MA, et al. Laparoscopic insertion with tip suturing, omentectomy, and ovariopexy improves lifespan of peritoneal dialysis catheters in children. J Laparoendosc Adv Surg Tech 2008; 18: 302-5.

12. Ovnat A, Dukhno O, Pinsk I, et al. The laparoscopic option in the management of peritoneal dialysis catheter revision. Surg Endosc 2002; 16: 968-9.

13. Wilson JA, Swartz RD. Peritoneoscopy in the management of catheter malfunction during continuous ambulatory peritoneal dialysis. Dig Dis Sci 1985; 30: 465-7.

14. Amerling A, Maele DV, Spivak H, et al. Laparoscopic salvage of malfunctioning peritoneal catheters. Surg Endosc 1997; 11: 249-52.

15. Nijhuis PHA, Smulders JF, Jakimowicz JJ. Laparoscopic introduction of a continuous ambulatory peritoneal dialysis (capd) catheter by a two-puncture technique. Surg Endosc 1996; 10: 676-9.

16. Dzielicki J, Grabowski A, Korlacki W. Optimizing the technique of laparoscopic splenectomy in children. Videosurgery and other miniinvasive techniques 2010; 5: 19-26.

17. Korlacki W, Dzielicki J, Grabowski A. Laparoscopic distal pancreatectomy in children with solid pseudopapillary tumour (Frantz 
tumour) - a report of two cases and review of the literature. Videosurgery and other miniinvasive techniques 2010; 5: 65-9.

18. Carrillo SA, Ghersi MM, Unger SW. Laparoscopic-assisted peritoneal dialysis catheter placement: a microinvasive technique. Surg Endosc 2007; 21: 825-9.

19. Goh YH. Omental folding: a novel laparoscopic technique for salvaging peritoneal dialysis catheters. Perit Dial Int 2008; 28: 626-31.

20. Yilmazlar T, Yavuz M, Ceylan H. Laparoscopic management of malfunctioning peritoneal dialysis catheters. Surg Endosc 2001; 15: 820-2. 